\title{
Prediction of Road Traffic Accidents in Jordan using Artificial Neural Network (ANN)
}

\author{
Khair S. Jadaan, Muaath Al-Fayyad, and Hala F. Gammoh \\ University of Jordan /Civil Engineering Department, Amman , Jordan \\ Email: kjadaan@hotmail.com, Civil.eng.muaath@gmail.com, Gammohhala@yahoo.com
}

\begin{abstract}
Highway related accidents are considered one of the most serious problems in the modern world as traffic accidents cause serious threat to human life worldwide. Jordan, a developing country, has high and growing level of traffic accidents resulting in more than 13000 fatalities between 1989 and 2012 with an average annual cost of over $\$ 500$ million. Prediction of future traffic accidents is therefore of utmost importance in order to appreciate the magnitude of the problem and speed up the decision making towards its alleviation. In this paper, a traffic accident prediction model was developed using the novel Artificial Neural Network (ANN) simulation with the aim of identifying its suitability for prediction of traffic accidents under Jordanian conditions. The results demonstrated that the estimated traffic accidents, based on sufficient data, are close enough to actual traffic accidents and thus are reliable to predict future traffic accidents in Jordan.
\end{abstract}

Index Terms - traffic accidents, neural network, developing countries, regression, prediction models

\section{INTRODUCTION}

Traffic accidents cause serious threat to human life worldwide. According to the World Health Organization (WHO), more than 1.2 million people die each year in motor vehicle accidents and more than 50 millions are injured worldwide. Jordan, as one of the developing countries, has high level of traffic accidents where there were more than 13000 fatalities between the years 19892012 calling for the need to determine the current and future magnitude of the epidemic in order to take necessary actions to curb this ever growing problem.

Extensive research has been carried out into the prediction of traffic accidents in both developed and developing countries using various statistical techniques. However, the numerous variables and complex relationships between the characteristics of the various traffic elements require analytical techniques other than traditional. A recent approach to analyze these relationships is the artificial neural networks (ANN) which has been proposed and employed successfully by many scientists as an alternative to the conventional regression approach in forecasting time series pertaining to complex atmo spheric and environmental phenomena. This paper presents and discusses the development of a

Manuscript received January 2, 2014; revised March 23, 2014. prediction model for estimating future traffic accidents in Jordan using the ANN approach.

\section{ARTIFICIAL NEURAL NETWORKS AND THEIR APPLICATIONS}

Artificial Neural Network is a sub-domain of artificial intelligence system which has been used recently to solve wide variety of civil engineering problems. A neural network is a data-modeling tool and an information processing paradigm that represents complex relationships in a manner similar to the human brain. ANNs are known to be universal function approximators and are capable of exploiting nonlinear relationships between variables.

Neural networks are a wide class of flexible nonlinear regression and discriminate models, data reduction models, and nonlinear dynamical systems. They consist of an often large number of "neurons," i.e. simple linear or nonlinear computing elements, interconnected in often complex ways and often organized into layers [1]. The key element of this paradigm is the novel structure of the information processing system. It is composed of:

- Highly interconnected processing elements (neurons. Each neuron has a value, weight and bias(constant) where the neuron's net input is the value of the neuron multiply by the weight plus the bias.

- Layers composed of an input layer which contains the data to be classified by the network (independent variables), one or more hidden layers which do the processing, and an output layer which contains the desired output ( dependent variable). Each layer consists of neurons connected to every other neuron in the previous layer by a link that represents the weight. An example of an ANN with its various layers is shown in "Fig. 1".[2]

- Activation functions: These are also called transfer functions that define the mappings from inputs to hidden nodes and from hidden nodes to output(s), respectively.[3] Artificial Neural Networks have been utilized successfully in solving engineering problems related to classification, prediction, and function approximation. In the transportation area, ANN has many applications and when applied to predict speed, for example, McFadden etal [4] found it to offer predictive power superior to 
those of regression models. This is mainly because of their ability to model non-linearity, and flexibility with large complex data sets.

- Further applications include the work of Shoukry [5] who used the ANNs in classification of severity levels of accidents and reported various applications of ANN in the transportation field especially in the traffic safety area.

- Chiou [6] employed ANN to develop an expert system for the appraisal of two-car accidents, Xiangzheng $\mathrm{Xu}$ [7] applied the ANNs technique to evaluate traffic safety in China, and Wenhui [8] researched the evaluation of safety in traffic accident scene based on ANN.

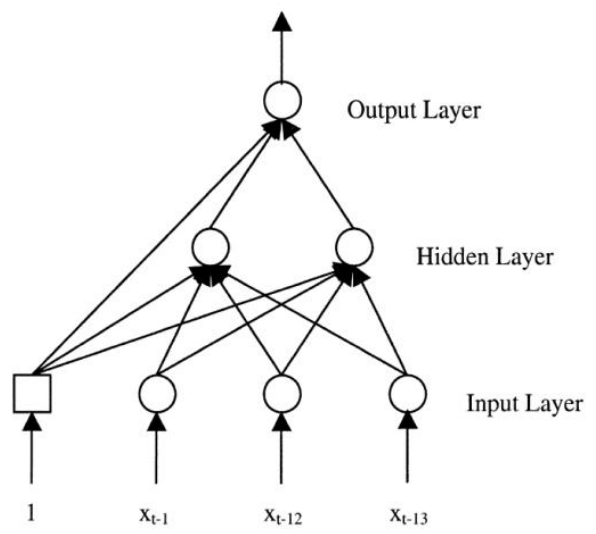

Figure 1. Typical layers in neural networks

\section{DEVELOPMENT OF ANN PREDICTION MODEL}

Developing the ANN model for accident prediction involved a sequence of steps as follows:

1.Data collection phase which included the following input data:

- V: number of registered vehicles

- P: population

- L: total length of paved roads

- $\mathrm{G}$ : the gross domestic product

The data is divided into three sets; training data.(about $70 \%$ of the total data), validation data (about $15 \%$ of the total data), and testing data (about $15 \%$ of the total data). Training, validation and testing of the network was performed using MATLAB. The statistical analysis was performed using the SPSS statistical software, and the Selected transfer functions were

- Input hidden layer : Tan-sigmoid transfer function

- Output hidden layer : Linear transfer function

The training process includes the following operations:

- Setting initial values for weights

- Evaluating the output based on initial weights.

- Measuring the error (mean square error or any function to calculate the error)

- Adjusting the weights using rate of learn(usually small value such as 0.01 )

- The weights continue to be modified as each error is computed. If the network is capable and the learning rate is set correctly, the error is eventually driven to zero.
- In the validation phase, no adjustment occurs to the weights. Validation is necessary to measure the performance of the network model where the predicted values are compared with the actual as given by the validation data. This process can be integrated with training process to improve the performance of the model

- Through the testing process, the predicted values are compared with the input values using testing data that was not used in training or validation process. Again, no adjustment occurs to the weights

- The architecture of the ANN was as shown in "Fig. 2". [9]

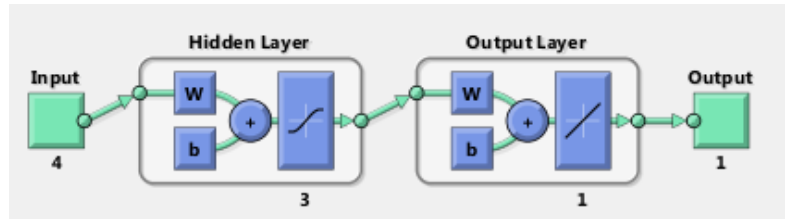

Figure 2. Architecture of the ANN

TABLE I. NEURAL NETWORK ALTERNATIVES

\begin{tabular}{|l|l|l|l|l|l|}
\hline $\begin{array}{l}\text { Model } \\
\text { No. }\end{array}$ & $\begin{array}{l}\text { Number } \\
\text { of hidden } \\
\text { layers }\end{array}$ & $\begin{array}{l}\mathrm{r}^{\mathrm{a}} \\
\text { (Training) }\end{array}$ & $\begin{array}{l}\mathrm{r} \\
\text { (Testing) }\end{array}$ & $\begin{array}{l}\mathrm{r} \\
\text { (Validation) }\end{array}$ & $\mathrm{R}^{2}$ \\
\hline 1 & 1 & 0.9972 & 0.9960 & 0.9998 & 0.986 \\
\hline 2 & 2 & 0.9995 & 0.9913 & 0.9984 & 0.987 \\
\hline 3 & 3 & 0.9984 & 0.9814 & 0.9966 & 0.989 \\
\hline $\mathbf{4}$ & $\mathbf{3}^{\mathbf{b}}$ & $\mathbf{0 . 9 9 5 0}$ & $\mathbf{0 . 9 9 9 9 9}$ & $\mathbf{0 . 9 9 9 4}$ & $\mathbf{0 . 9 9 2}$ \\
\hline
\end{tabular}

b. three hidden layers with several training cycles

TABLE II. ACTUAL AND PREDICTED NUMBER OF ACCIDENTS USING ANN

\begin{tabular}{|l|l|l|l|}
\hline Year & \multicolumn{1}{|l|}{ Actual } & Predicted & Residual \\
\hline 1990 & 17838 & 25262.53 & -7424.53 \\
\hline 1991 & 18756 & 19977.13 & -1221.13 \\
\hline 1992 & 20970 & 17948.62 & 3021.383 \\
\hline 1993 & 24799 & 20034.64 & 4764.365 \\
\hline 1994 & 26837 & 26820.84 & 16.16152 \\
\hline 1995 & 28970 & 29761.88 & -791.879 \\
\hline 1996 & 33784 & 30965.42 & 2818.581 \\
\hline 1997 & 39005 & 34767.66 & 4237.336 \\
\hline 1998 & 43343 & 39702.99 & 3640.009 \\
\hline 1999 & 50330 & 44307.13 & 6022.865 \\
\hline 2000 & 52796 & 54230.03 & -1434.03 \\
\hline 2001 & 52662 & 55018.03 & -2356.03 \\
\hline 2002 & 52913 & 57601.76 & -4688.76 \\
\hline 2003 & 62115 & 62542.11 & -427.112 \\
\hline 2004 & 70266 & 69560.79 & 705.2115 \\
\hline 2005 & 83129 & 85324.17 & -2195.17 \\
\hline 2006 & 98055 & 100570.1 & -2515.1 \\
\hline 2007 & 110630 & 110845.4 & -215.371 \\
\hline 2008 & 101066 & 109537.3 & -8471.32 \\
\hline 2009 & 122793 & 125060.2 & -2267.2 \\
\hline 2010 & 139396 & 139254.2 & 141.7603 \\
\hline 2011 & 142588 & 151803.8 & -9215.79 \\
\hline & & & \\
\hline
\end{tabular}

IV. RESULTS 
The Neural Networks allow the development of different alternatives by changing the number of hidden layers. Four alternative models, with different number of hidden layers, were considered and Table I summarizes the results.

Model 4 was found to be the best model with the highest coefficient of determination $\left(\mathrm{R}^{2}=0.992\right)$. A comparison between the actual and the predicted values using model 4 produced the results shown in Table II. The results were found to be very satisfactory with relatively small residuals especially in recent years where more reliable data bases are available through using more advanced data compilation techniques. The various ANN model outputs are displayed in "Fig. 3".
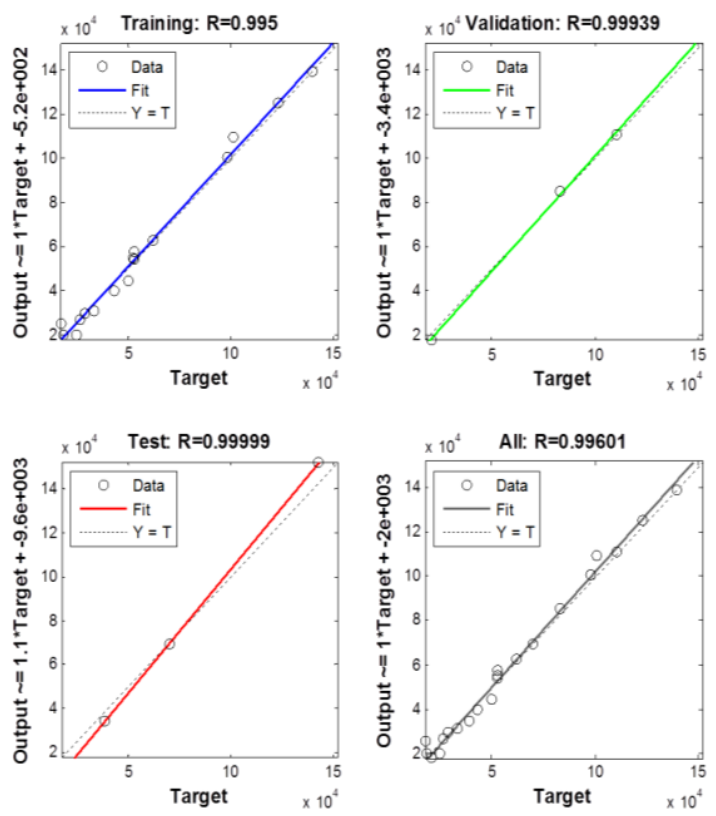

Figure 3. ANN output

\section{CONCLUSION}

Road traffic accident in Jordan constitutes a serious problem and prediction of its future magnitude using reliable approaches has become a necessity. Artificial Neural Networks (ANN) is a novel approach which proved to be successful in solving engineering problems and researchers found it to offer productive power superior to those of traditional regression models.

An accident prediction model was developed using the ANN approach through analyzing the relationship between accidents and parameters affecting them for which data were available. The model was validated and found to produce good results under Jordanian traffic conditions thus can be used with confidence to predict future traffic accidents on the national road network.

\section{REFERENCES}

[1] W. S. Sarle, "Neural networks and statistical models," in Proc. Nineteenth Annual SAS Users Group International Conference, , NC, USA, April 1994

[2] S. D. Balkina, et al., "Automatic neural network modeling for univariate time series," International Journal of Forecasting, vol. 16, no. 4, pp. 509-515, 2000.

[3] Neural Network Toolbox, User's Guide, Mark Hudson Beale, Martin T. Hagan, Howard B. Demuth, 2013.

[4] J. McFadden, W. T. Yang, and R. Durrans, "Application of ANN to predict speeds on two-lane rural highways," Transportation Research Record 1751, pp. 9-17, 2000.

[5] F. N. Shoukry, "Artificial neural network in classification of severity levels in crashes with guardrail," Masters of Science Thesis, West Virginia University, USA, 2005.

[6] Y. C. Chiou, "An artificial neural network- based expert system for the appraisal of two-car crash accidents," Department of Traffic and Transportation Engineering and Management, Feng Chia University, Taiwan, July 2006.

[7] X. Xu, B. Chen, and F Gan, "Traffic safety evaluations based on grey systems theory and neural networks," in Proc. WRI World Congress on Computer Science and Information Engineering, vol. 5, 2009, pp. 603-607.

[8] Z. Wenhui, "Safety evaluation of traffic accident scene based on artificial neural network," ICICTA, China, vol. 1, Oct. 2009, pp. 408-410.

[9] Personal Communication with Mosa AL-Akhras, IT Department, University of Jordan, 2013.

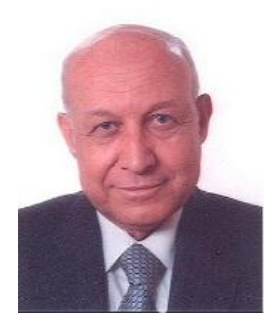

Prof. Dr. Khair Jadaan born in 1948 earned his Ph.D. degree in traffic engineering and planning in 1975 from the University of Bradford, U.K.

Khair working experience covers a variety of positions in both private and public sectors in various developed and developing countries including New Zealand, Germany, U.K., U.S.A., Kuwait, Iraq and Jordan. $\mathrm{He}$ is currently a PROFESSOR of transportation engineering at the University of Jordan. He published over 100 papers in international journals and conferences.

Prof. Jadaan is a Fellow of IHTE (U.K), member of IPENZ(New Zealand), member of ASCE (USA) and Jordan Engineers Association and been awarded a number of honoraries.

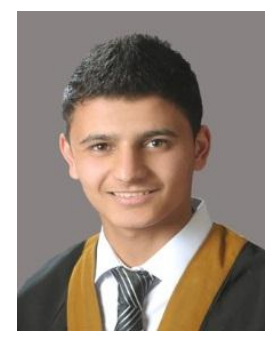

Mr Muaath Al-Fayyad was born in 1991and earned his B.Sc. degree in Civil Engineering in June 2013 from the University of Jordan. He had his training with Concord Contracting Company and is currently working as a Civil Engieer with Dar Al-Handasa, one of the largest consulting firms in the region.During his undergrauate studies, Moath produced reports on several civil engineering projects including transportation.

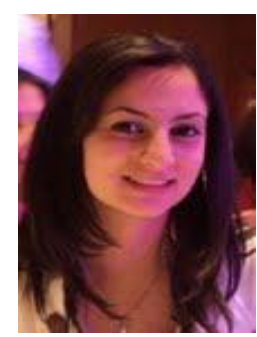

Miss Hala Gammoh born in 1992 is an undergraduate student at the civil engineering department, University of Jordan. She is a MEMBER of the junior research team of the department. Her research interests include traffic safety and environmental impact of transport. Currently she is working on a research project into the social and economic impacts of transport. 